\title{
GIS Technique Based Spatio Temporal Variation Study of Ground Water Quality Parameters of SIDCUL-Pantnagar, India
}

\author{
Dheeraj Kumar ${ }^{1 *}$, Yogendra Kumar², Mohan lal ${ }^{3}$, Mahima Bora ${ }^{4}$, \\ Sakshi Gautam ${ }^{5}$ and Sidra Souban ${ }^{6}$
}

Department of Irrigation \& Drainage Engineering, College of Technology, G.B. Pant. University of Agriculture \& Technology, Pantnagar, Dist-Udham Singh Nagar-263145 (U.K), India

*Corresponding author

\section{A B S T R A C T}

\section{Keywords}

Physcio-chemical, Thematic maps, GIS and WQI

Article Info

Accepted:

18 March 2020 Available Online:

10 April 2020
The physico-chemical ex-situ study was conducted for 21 groundwater sample of SIDCUL - Udham Singh Nagar district, Uttarakhand. The various physicochemical parameters such as $\mathrm{pH}$, Electrical Conductivity (EC), Total Dissolved Solids (TDS), Calcium $\left(\mathrm{Ca}^{2+}\right)$, Magnesium $\left(\mathrm{Mg}^{2+}\right)$, Sodium $\left(\mathrm{Na}^{+}\right)$, Potassium $\left(\mathrm{K}^{+}\right)$, Bicarbonate $\left(\mathrm{HCO}_{3}\right)$, Carbonate $\left(\mathrm{CO}_{3}\right)$, Chloride $\left(\mathrm{Cl}^{-}\right)$, Turbidity and Nitrate $\left(\mathrm{NO}_{3}\right)$ were determined using standard procedures. BIS water quality standard for drinking purpose was used to compare the results. BIS standard was used to generate spatial distribution thematic maps of groundwater quality parameters for drinking purpose. $\mathrm{pH}$ of the study area showed that sixteen ground water samples were found unsuitable for drinking purpose. WQI thematic maps indicated the groundwater of all sites were found excellent over an area of $4.58 \mathrm{~km}^{2}$ with $14.07 \%$ area, good over an area of $18.24 \mathrm{~km}^{2}$ with $56.03 \%$ area, poor over an area of $6.33 \mathrm{~km}^{2}$ with $19.44 \%$ area, very poor over an area of $1.50 \mathrm{~km}^{2}$ with $4.62 \%$ area, unsuitable over an area of $1.89 \mathrm{~km}^{2}$ with $5.81 \%$ area.

\section{Introduction}

Water is one of the most important compounds that profoundly influence life. Human population largely depends on water resources for their survival and development. Water is a key element for day-to-day human, agricultural and life activities. Since time immemorial, human society has continuously been in interaction with nature for agriculture.
Getting back to this gradual evolution we see that initially water was largely needed for irrigating crops. Public health, industry and recreation claimed a very small percentage of the total water supply.

The requirements of water increased with time to meet the requirement of more food with increasing population. As water is being used for different purposes, its quality 
becomes an important and crucial issue for being considered. Water quality means whether the nature of water is suitable for use in various fields like for irrigating, for industries or for drinking. For e.g., if water has more chloride or sulphide content, then it is not suitable and good for cleaning purposes and is thus said to be of poor quality.

Groundwater is the water located beneath Earth's surface in soil pore spaces and in the fractures of rock formation. The crucial role which groundwater plays is as a decentralized source of drinking water for millions rural and urban families. It is generally estimated that approximately one thirds of the world's population uses groundwater for drinking purposes. According to some other estimates, it accounts for nearly 80 per cent of the rural domestic water needs, and 50 per cent of the urban water needs in India (Alam et al., 2009). Groundwater is generally less susceptible to contamination and pollution when compared to surface water bodies. In India, where groundwater is used intensively for irrigation and industrial purposes, a variety of land and water-based human activities are causing pollution of this precious resource, its over-exploitation is causing aquifer contamination in certain instances, while in others its unscientific development with insufficient knowledge of groundwater flow dynamic and geohydrochemical processes has

Water quality is determined by the chemical, physical and biological parameters of water. It is a measure of the state of the water with respect to the necessities of human needs or purposes (Abbasi and Abbasi, 2012). The use of GIS techniques is vital in testing and improving the groundwater contamination risk assessment methods. For any city, a groundwater quality map is important to evaluate the water safeness for drinking and irrigation purposes and also as a precautionary indication of potential environmental health problems. Keeping these facts in view the present study has been conducted $\mathrm{n}$ to evaluate spatio temporal variability of groundwater quality for drinking purpose.

\section{Materials and Methods}

\section{Descriptions of study area and water sample collection}

The study area is located in SIDCUL (State Industrial Development Corporation Uttarakhand Limited) near Rudrapur, Udham Singh Nagar district. The geographical area of the district is $3055 \mathrm{~km}^{2}$. The study area is located between $28^{0} 57^{\prime} 40.5432$ ' $\mathrm{N}$ to $29^{\circ} 02$ ' 21.0948 "N latitude and laterally extends between $79^{0} 23^{\prime} 48.5406^{\prime \prime} \mathrm{E}$ to $79^{0} 27^{\prime} 10.2528^{\prime \prime} \mathrm{E}$ longitude. The district is bounded by Nainital and Champawat districts of Uttarakhand on the North, Moradabad, Rampur, Bareilly and Pilibhit districts of Uttar Pradesh on West and Nepal on the East. The region is being polluted to a great extent by the effluents discharged from various industries situated at SIDCUL (Pantnagar). The location maps of sampling sites are shown in Figure 1 respectively and the location of groundwater sampling sites is represented in Table 1. The water samples for the physico-chemical analysis were collected on 26-April-2019 from shallow hand pumps of 21 locations of the study area. Samples were collected using plastic bottles and were kept in the incubator at a temperature of so that no or minimum changes occur in the physico-chemical properties of the water samples.

\section{Physico-chemical analysis}

The physic-chemical parameters such as $\mathrm{pH}$, Electrical Conductivity (EC), Total Dissolved Solids (TDS), Calcium $\left(\mathrm{Ca}^{2+}\right)$, Magnesium 
$\left(\mathrm{Mg}^{2+}\right)$, Sodium $\left(\mathrm{Na}^{+}\right)$, Potassium $\left(\mathrm{K}^{+}\right)$, Bicarbonate $\left(\mathrm{HCO}_{3}\right)$, Carbonate $\left(\mathrm{CO}_{3}\right)$, Chloride $\left(\mathrm{Cl}^{-}\right)$, Turbidity and Nitrate $\left(\mathrm{NO}_{3}\right)$ were determined using standard methods. The methods used for estimation of various physic-chemical parameters are given in Table 2.

\section{Water quality spatial index}

Weighted arithmetic water quality index (WAWQI) method categorized the water quality as a grade of purity on the basis of normally measured water quality variables. WAWQI method had been used by the various scientists (Chauhan and Singh, 2010; Chowdhury et al., 2010; Balan et al., 2012). In the present study WQI is estimated using the following equation:

$\mathrm{WQI}=\frac{\sum_{\mathrm{i}=1}^{\mathrm{n}} \mathrm{Q}_{\mathrm{i}} \mathrm{W}_{\mathrm{i}}}{\sum_{\mathrm{i}=1}^{\mathrm{n}} \mathrm{w}_{\mathrm{i}}}$

The quality rating scale $\left(\mathrm{Q}_{\mathrm{i}}\right)$ for each parameter is calculated by using this expression:

$Q=\frac{V_{\mathrm{a}}-\mathrm{V}_{\mathrm{i}}}{\mathrm{s}_{\mathrm{i}}-\mathrm{V}_{\mathrm{o}}} \times 100$

Where,

$V_{a}=$ estimated concentration of $i^{\text {th }}$ parameter in the analyzed water;

$V_{i}=$ ideal value of $\mathrm{i}^{\text {th }}$ parameter;

$V_{0}=0($ except $\mathrm{pH}=7.0)$ and

$S_{i}=$ recommended standard value of $\mathrm{i}^{\text {th }}$ parameter.

The unit weight $\left(\mathrm{W}_{\mathrm{i}}\right)$ for each water quality parameter was calculated using following formula:

$$
W_{i=\frac{K}{s_{i}}}
$$

where, $\mathrm{K}$ is the proportionality constant calculated by using the following equation:

$$
K=\frac{1}{\sum_{\mathrm{i}=0}^{\mathrm{n}} \mathrm{S}_{\mathrm{i}}}
$$

The detailed procedure and classes of water quality index based on the weighted arithmetic WQI is shown in Table 2 and Figure 2.

\section{Groundwater quality spatial distribution thematic maps}

On the basis of results of analysis different water quality parameter thematic maps (as per BIS standard) and WQI thematic maps for SIDCUL (Pantnagar) were prepared in GIS environment using Arc GIS 10.2 software. Inverse Distance Weighted Moving Average (IDWMA) interpolation method was used to prepare different thematic map of ground water quality parameters under GIS environment. The detailed procedure for water quality thematic map and water quality index map are shown in Fig. 3 and 4.

\section{Results and Discussion}

$\begin{aligned} & \text { Suitability of } \\ & \text { characteristics of water sample for } \\ & \text { drinking purpose }\end{aligned}$

All the water samples analyzed during premonsoon period and the results revealed that a minimum $\mathrm{pH}$ value of 5.9 was observed at Indra Chaurahaand maximum $\mathrm{pH}$ value of 6.6 was observed at Nand Vihar Colony. The average value was observed as 6.25. All the samples analyzed except the sample nearby Chief Medical Officer Office road, Nand Vihar Colony, Model Medicinal Plants Garden, Pracheen Van Shakti and Haldi Attariya Mandir were found below the BIS permissible limit of $\mathrm{pH}(6.5-8.5)$ and were considered unsuitable for drinking purpose. A minimum EC value of $346 \mu \mathrm{S} / \mathrm{cm}$ was 
observed at Haldi Attariya Mandir and maximum value of $1162 \mu \mathrm{S} / \mathrm{cm}$ was observed at opposite to Tehsil. The average value was observed as $754 \mu \mathrm{S} / \mathrm{cm}$. A minimum TDS value of $180 \mathrm{mg} / \mathrm{l}$ was observed at Haldi Attariya Mandir, Jagatpura and maximum TDS value of $552 \mathrm{mg} / \mathrm{l}$ was observed at Indra Chauraha. The average value was observed as $366 \mathrm{mg} / \mathrm{l}$. All the samples analyzed except opposite to Tehsil, Gangapur road, Indra chauraha were found within the BIS permissible limit of TDS $(<500 \mathrm{mg} / \mathrm{l})$ and were considered suitable for drinking purpose. A minimum total acidity value of 30 $\mathrm{mg} / \mathrm{l}$ was observed nearby Chief Medical Officer Office road and a maximum total acidity value of $132.5 \mathrm{mg} / \mathrm{l}$ was observed at Indra Chauraha. The average value was observed as $81.25 \mathrm{mg} / \mathrm{l}$. A minimum temperature of $24.4^{0} \mathrm{C}$ was observed Haldi Attariya Mandir Jagatpura nearby and maximum temperature of $32^{\circ} \mathrm{C}$ at Balaji sweet shop Police chauki Rampura. The average value was observed as $28.2^{\circ} \mathrm{C}$. A minimum chloride value of $26.98 \mathrm{mg} / \mathrm{l}$ was observed at Matkota TDC main road and maximum chloride value of $99.4 \mathrm{mg} / \mathrm{l}$ was observed at Indra Chauraha. The average value was observed as $63.19 \mathrm{mg} / \mathrm{l}$. All the samples analyzed were found below the BIS permissible limit of chloride (250-1000 mg/l) and were considered suitable for drinking purpose. A minimum calcium hardness value of $100.07 \mathrm{mg} / \mathrm{l}$ was observed nearby Chief Medical Officer Office road and a maximum calcium value of $250.19 \mathrm{mg} / \mathrm{l}$ was observed at opposite to Tehsil. The average value was observed as $175.13 \mathrm{mg} / \mathrm{l}$. A minimum magnesium hardness value of $169.9 \mathrm{mg} / \mathrm{l}$ was observed at MatkotaTDC main road and maximum magnesium hardness value of $469.8 \mathrm{mg} / \mathrm{l}$ was observed near Hanuman Mandir Bus station. The average value was observed as $319.85 \mathrm{mg} / \mathrm{l}$. A minimum total hardness value of $290 \mathrm{mg} / \mathrm{l}$ was observed at Matkota TDC main road and maximum total hardness value of $720 \mathrm{mg} / \mathrm{l}$ was observed at opposite to Tehsil. The average value was observed as $505 \mathrm{mg} / \mathrm{l}$. A minimum sodium value of $1.2 \mathrm{meq} / \mathrm{l}$ was observed nearby Chief Medical Officer Office road and maximum sodium value of $15.2 \mathrm{meq} / \mathrm{l}$ was observed at Indra Chauraha. The average value was observed as $8.2 \mathrm{meq} / \mathrm{l}$. A minimum potassium value of $0.3 \mathrm{meq} / \mathrm{l}$ was observed nearby in front of Pal mutton shop and maximum potassium value of $3.9 \mathrm{meq} / \mathrm{l}$ was observed at Indra Chauraha. The average value was observed as $2.1 \mathrm{meq} / \mathrm{l}$. A minimum free $\mathrm{CO}_{2}$ value was observed nearby Matkota TDC main road whose value was $17.6 \mathrm{mg} / \mathrm{l}$ and maximum free $\mathrm{CO}_{2}$ value of $101.2 \mathrm{mg} / \mathrm{l}$ was observed at Tehsil Rudrapur. The average value was observed as $59.3 \mathrm{mg} / \mathrm{l}$. A minimum nitrate value of $0.52 \mathrm{mg} / \mathrm{l}$ was observed at Mukesh road lines Fulsunga and maximum nitrate value of $0.69 \mathrm{mg} / \mathrm{l}$ was observed at Phoolbagh Centre. The average value was observed as $0.605 \mathrm{mg} / \mathrm{l}$. A minimum bicarbonate value of $45.15 \mathrm{mg} / \mathrm{l}$ was observed at Balaji sweet shop police chauki Rampura and maximum bicarbonate value of 141.55 $\mathrm{mg} / \mathrm{l}$ was observed at Pallavika nursery 18 Aavas Vikas. The average value was observed as $93.335 \mathrm{mg} / \mathrm{l}$. Carbonate concentration in all water sample was found zero. A minimum total alkalinity value of $225 \mathrm{mg} / \mathrm{l}$ was observed Haldi Attariya Mandir and maximum total alkalinity value of $545 \mathrm{mg} / \mathrm{l}$ was observed at opposite to Tehsil. The average value was observed as $385 \mathrm{mg} / \mathrm{l}$. A minimum turbidity value of 1 NTU was observed at Model Medicinal Plants Garden, J-Block, HRC, Matkota TDC main road, nearby Chief Medical Officer Office road, Pracheen Van Shakti Mandir, Pallavika nursery 18 Aavas Vikas Rudrapur, Hanuman Mandir Bus station, Divya traders, Phulsungi, Nand Vihar Colony and maximum turbidity value of 25 NTU was observed at Balaji sweet shop police chauki Rampura. The average value was observed as 13 NTU. All 
the samples analyzed except Police chauki Rampura, Indra chauraha and Haldi Seed Processing Plant were found within the BIS permissible limit of turbidity (10 NTU) and were considered suitable for drinking purpose. The results of various physiochemical parameters are given in Table 4.

\section{Spatio-temporal variations of groundwater quality parameters}

Using Arc GIS, the area wise distribution of various parameters for pre monsoon sampling are given in Table 5 and thematic map of spatial distribution of these parameters are shown from Figure 5 to 7 . TDS of groundwater samples was classified on the basis of BIS Standards. Figure 5 reveals that $98.59 \%$ of study area $\left(32.09 \mathrm{~km}^{2}\right)$ was found within the BIS permissible limit $(<500)$, and $1.406 \%$ of study area $\left(0.457 \mathrm{~km}^{2}\right)$ was found above the BIS permissible limit $(>500)$ during pre-monsoon period. $\mathrm{Ca}$ content of groundwater samples was classified on the basis of BIS Standards. Figure 6 reveals that $91.95 \%$ of study area $\left(29.93 \mathrm{~km}^{2}\right)$ was found below the BIS permissible limit (75-200 mg/l) and $8.04 \%$ of study area $\left(2.617 \mathrm{~km}^{2}\right)$ was found within the BIS permissible limit (75$200 \mathrm{mg} / \mathrm{l}$ ) during pre-monsoon period. $\mathrm{Mg}$ content of groundwater samples was classified on the basis of BIS Standards. Figure 7 reveals that $98.90 \%$ of study area $\left(32.19 \mathrm{~km}^{2}\right)$ was found within the BIS permissible limit (30-100 mg/l) and $10.977 \%$ of study area $\left(0.357 \mathrm{~km}^{2}\right)$ was found within the BIS permissible limit (30-100 mg/l) during pre-monsoon period.

Table.1 Location of ground water sampling sites

\begin{tabular}{|c|c|c|c|c|}
\hline Sites & Locations & Latitude & Longitude & $\begin{array}{l}\text { Altitude above } \\
\text { MSL }\end{array}$ \\
\hline 1 & Phoolbagh centre, near primary school & $29^{\circ} 01^{\prime} 15.82^{\prime \prime} \mathrm{N}$ & $79^{\circ} 28^{\prime} 19.24^{\prime \prime} \mathrm{E}$ & $234 \mathrm{~m}$ \\
\hline 2 & $\begin{array}{l}\text { Model Medicinal Plants Garden, Medicinal Plant } \\
\text { Research Centre Gate }\end{array}$ & $29^{\circ} 01^{\prime} 54.88^{\prime \prime} \mathrm{N}$ & $79^{\circ} 27^{\prime} 52.27^{\prime \prime} \mathrm{E}$ & $234 m$ \\
\hline 3 & Haldi Seed Processing Plant & $29^{\circ} 1 ' 52.62 " \mathrm{~N}$ & $79^{\circ} 26^{\prime} 49.80^{\prime \prime} \mathrm{E}$ & $231 \mathrm{~m}$ \\
\hline 4 & H-Block Haldi & $29^{\circ} 00^{\prime} 0.66^{\prime \prime} \mathrm{N}$ & $79^{\circ} 25^{\prime} 8.04^{\prime \prime} \mathrm{E}$ & $218 \mathrm{~m}$ \\
\hline 5 & I-Blocklabour shed farmstore & $29^{\circ} 0^{\prime} 45.30^{\prime \prime} \mathrm{N}$ & $79^{\circ} 26^{\prime} 10.14 " \mathrm{E}$ & $223 m$ \\
\hline 6 & J-Block Haldi labour shed (J-Block headquarters) & $29^{\circ} 2^{\prime} 11.40 " \mathrm{~N}$ & $79^{\circ} 25^{\prime} 29.52^{\prime \prime} \mathrm{E}$ & $228 \mathrm{~m}$ \\
\hline 7 & Shiv Mandir Pattharchatta & $29^{\circ} 2^{\prime} 11.40^{\prime \prime} \mathrm{N}$ & $79^{\circ} 25^{\prime} 2.88^{\prime \prime} \mathrm{E}$ & $229 \mathrm{~m}$ \\
\hline 8 & Matkota TDC main road & $29^{\circ} 00^{\prime} 31.62^{\prime \prime} \mathrm{N}$ & $79^{\circ} 24^{\prime} 1.14 " \mathrm{E}$ & $219 m$ \\
\hline 9 & Chief Medical Officer Office road & $29^{\circ} 00^{\prime} 1.02^{\prime \prime} \mathrm{N}$ & 79²3'29.16"E & $217 \mathrm{~m}$ \\
\hline 10 & Infront of Pal mutton shop (Attariya road) & $28^{\circ} 59^{\prime} 31.02^{\prime \prime} \mathrm{N}$ & $79^{\circ} 24^{\prime} 2.52^{\prime \prime} \mathrm{E}$ & $215 \mathrm{~m}$ \\
\hline 11 & Haldi Attariya Mandir, Jagatpura & $28^{\circ} 59^{\prime} 31.98^{\prime \prime} \mathrm{N}$ & $79^{\circ} 24^{\prime} 27.42^{\prime \prime} \mathrm{E}$ & $216 m$ \\
\hline 12 & Raj auto service Attariya road & $28^{\circ} 59^{\prime} 23.16^{\prime \prime} \mathrm{N}$ & $79^{\circ} 24^{\prime} 39.54^{\prime \prime} \mathrm{E}$ & $215 \mathrm{~m}$ \\
\hline 13 & Pracheen Van Shakti Mandir & $28^{\circ} 59^{\prime} 24.12^{\prime \prime} \mathrm{N}$ & $79^{\circ} 25^{\prime} 30.54 " \mathrm{E}$ & $218 \mathrm{~m}$ \\
\hline 14 & Mukesh road lines, Fulsunga & $28^{\circ} 59^{\prime} 24.12^{\prime \prime} \mathrm{N}$ & $79^{\circ} 25^{\prime} 30.54^{\prime \prime} \mathrm{E}$ & $218 \mathrm{~m}$ \\
\hline 15 & Pallavika nursery 18 Aavas Vikas Rudrapur & $28^{\circ} 59^{\prime} 15.78^{\prime \prime} \mathrm{N}$ & $79^{\circ} 24^{\prime} 1.86^{\prime \prime} \mathrm{E}$ & $215 \mathrm{~m}$ \\
\hline 16 & $\begin{array}{l}\text { Hanuman Mandir, in front of Ramleela } \\
\text { Committee Bus station Rudrapur }\end{array}$ & $28^{\circ} 58^{\prime} 39.72^{\prime \prime} \mathrm{N}$ & $79^{\circ} 24^{\prime} 4.62^{\prime \prime} \mathrm{E}$ & $215 \mathrm{~m}$ \\
\hline 17 & Tehsil Rudrapur Gangapur road & $28^{\circ} 58^{\prime} 27.48^{\prime \prime} \mathrm{N}$ & $79^{\circ} 24^{\prime} 32.10^{\prime \prime} \mathrm{E}$ & $214 m$ \\
\hline 18 & Divya traders, Phulsungi & $28^{\circ} 58^{\prime} 32.34^{\prime \prime} \mathrm{N}$ & $79^{\circ} 25^{\prime} 10.98^{\prime \prime} \mathrm{E}$ & $213 \mathrm{~m}$ \\
\hline 19 & Nand Vihar Colony & $28^{\circ} 58^{\prime} 32.10^{\prime \prime} \mathrm{N}$ & $79^{\circ} 25^{\prime} 26.16^{\prime \prime} \mathrm{E}$ & $214 \mathrm{~m}$ \\
\hline 20 & Balaji sweet shop, near Police Chauki Rampura & $28^{\circ} 58^{\prime} 5.94 " \mathrm{~N}$ & $79^{\circ} 24^{\prime} 3.36 " \mathrm{E}$ & $213 m$ \\
\hline 21 & Indra Chauraha & $28^{\circ} 58^{\prime} 17.16^{\prime \prime} \mathrm{N}$ & $79^{\circ} 23^{\prime} 41.82^{\prime \prime} \mathrm{E}$ & $213 m$ \\
\hline
\end{tabular}


Table.2 Methods used for estimation of physiochemical parameters

\begin{tabular}{|c|c|c|c|}
\hline S.No. & Parameters & Method & References \\
\hline 1 & $\mathrm{pH}$ & $\begin{array}{l}\text { Using Glass } \\
\text { Electrode } \mathrm{pH} \text { meter }\end{array}$ & Jackson (1973) \\
\hline 2 & Electrical Conductivity & Using EC meter & Wilcox (1950) \\
\hline 3 & Total Dissolved Solids & Using TDS meter & Singh and Kalra (1975) \\
\hline 4 & $\begin{array}{l}\text { Calcium and } \\
\text { Magnesium }\end{array}$ & EDTA titration & $\begin{array}{l}\text { Cheng \& Bray (1951) } \\
\text { and Diehl et al., (1950) }\end{array}$ \\
\hline 5 & Sodium & $\begin{array}{l}\text { Flame Photometric } \\
\text { method }\end{array}$ & Toth et al., (1948) \\
\hline 7 & Potassium & $\begin{array}{l}\text { Flame Photometric } \\
\text { method }\end{array}$ & $\begin{array}{l}\text { Stanford and English } \\
\text { (1949) }\end{array}$ \\
\hline 8 & $\mathrm{CO}_{3}$ and $\mathrm{HCO}_{3}$ & $\begin{array}{l}\text { Titration with } \\
\text { standard } \mathrm{H}_{2} \mathrm{SO}_{4}\end{array}$ & A.O.A.C. (1950) \\
\hline 9 & Chloride & $\begin{array}{l}\text { Silver Nitrate } \\
\text { method }\end{array}$ & A.O.A.C.(1950) \\
\hline 10 & Sulphate & Titrimetric method & Munger et al., (1950) \\
\hline
\end{tabular}

Table.3 Water quality rating by weighted arithmetic index method

\begin{tabular}{|c|c|c|}
\hline WQI value & Rating of water quality & Grading \\
\hline$\leq \mathbf{2 5}$ & Excellent & $\mathrm{A}$ \\
\hline $\mathbf{2 5}-\mathbf{5 0}$ & Good & $\mathrm{B}$ \\
\hline $\mathbf{5 0}-\mathbf{7 5}$ & Poor & $\mathrm{C}$ \\
\hline $\mathbf{7 5}-\mathbf{1 0 0}$ & Very poor & $\mathrm{D}$ \\
\hline $\mathbf{> 1 0 0}$ & Unsuitable & E \\
\hline
\end{tabular}

\section{Water quality index}

For all the water samples analysed during premonsoon period, a minimum WQI value of 9.22 was observed at Matkota TDC main road of SBI and maximum WQI value of 245.71 was observed at Balaji sweet shop, Police chauki Rampura (Kachahari purana court). Using Arc GIS, Groundwater samples collected from all sites were classified on the basis of WQI and Figure 8 reveals that WQI was excellent over an area of $4.58 \mathrm{~km}^{2}$ with $14.07 \%$ area, good over an area of $18.24 \mathrm{~km}^{2}$ with $56.03 \%$ area, poor over an area of 6.33 $\mathrm{km}^{2}$ with $19.44 \%$ area, very poor over an area of $1.50 \mathrm{~km}^{2}$ with $4.62 \%$ area, unsuitable over an area of $1.89 \mathrm{~km}^{2}$ with $5.81 \%$ area during pre-monsoon period. 
Table.4 Physico-chemical properties of ground water sample

\begin{tabular}{|c|c|c|c|c|c|c|c|c|c|c|c|c|c|c|c|c|c|c|c|c|c|c|}
\hline $\begin{array}{l}\text { S. } \\
\text { No }\end{array}$ & $\begin{array}{c}\text { Location } \\
\text { Parameter }\end{array}$ & Site-1 & $\begin{array}{l}\text { Site- } \\
2\end{array}$ & Site-3 & $\begin{array}{l}\text { Site- } \\
4\end{array}$ & $\begin{array}{l}\text { Site- } \\
5\end{array}$ & $\begin{array}{l}\text { Site- } \\
6\end{array}$ & $\begin{array}{l}\text { Site- } \\
7\end{array}$ & $\begin{array}{l}\text { Site- } \\
8\end{array}$ & $\begin{array}{l}\text { Site- } \\
9\end{array}$ & $\begin{array}{l}\text { Site- } \\
10\end{array}$ & $\begin{array}{c}\text { Site- } \\
11\end{array}$ & $\begin{array}{l}\text { Site- } \\
12\end{array}$ & $\begin{array}{l}\text { Site- } \\
13\end{array}$ & $\begin{array}{l}\text { Site- } \\
14\end{array}$ & $\begin{array}{l}\text { Site- } \\
15\end{array}$ & $\begin{array}{l}\text { Site- } \\
16\end{array}$ & Site-17 & $\begin{array}{l}\text { Site- } \\
18\end{array}$ & $\begin{array}{l}\text { Site- } \\
19\end{array}$ & Site-20 & Site-21 \\
\hline 1. & Temperature $\left({ }^{0} \mathrm{C}\right)$ & 24.8 & 24.2 & 26.8 & 24.6 & 26.2 & 24.2 & 32 & 25 & 24.8 & 26 & $24.4^{0}$ & 26.4 & 26.8 & 28 & 24.8 & 25.8 & 26.8 & 27.6 & 28 & 29.4 & 26.2 \\
\hline 2. & $\mathrm{pH}$ & 6 & 6.5 & 6.3 & 6.1 & 6.2 & 6.2 & 6.4 & 6.4 & 6.5 & 6.2 & 6.6 & 6.3 & 6.5 & 6.3 & 6.1 & 6.4 & 6 & 6.4 & 6.6 & 6 & 5.9 \\
\hline 3. & $\mathrm{EC}(\mu \mathrm{S} / \mathrm{cm})$ & 796 & 635 & 736 & 621 & 558 & 562 & 426 & 361 & 358 & 489 & 346 & 561 & 433 & 561 & 855 & 566 & 1162 & 533 & 402 & 888 & 1045 \\
\hline 4. & TDS (mg/l) & 419 & 419 & 391 & 332 & 295 & 299 & 228 & 190 & 192 & 261 & 180 & 297 & 233 & 297 & 449 & 310 & 611 & 281 & 209 & 482 & 552 \\
\hline 5. & Ca hardness (mg/l) & $\begin{array}{c}190.1 \\
5\end{array}$ & $\begin{array}{c}190.1 \\
5\end{array}$ & 240.19 & $\begin{array}{c}190.1 \\
5\end{array}$ & $\begin{array}{c}150.1 \\
1\end{array}$ & $\begin{array}{c}150.1 \\
1\end{array}$ & $\begin{array}{c}120.0 \\
9\end{array}$ & $\begin{array}{c}120.0 \\
9\end{array}$ & $\begin{array}{c}100.0 \\
7\end{array}$ & $\begin{array}{c}120.0 \\
9\end{array}$ & $\begin{array}{c}140.1 \\
1\end{array}$ & $\begin{array}{c}140.1 \\
1\end{array}$ & $\begin{array}{c}140.1 \\
1\end{array}$ & $\begin{array}{c}130.1 \\
0\end{array}$ & $\begin{array}{c}220.1 \\
7\end{array}$ & $\begin{array}{c}180.1 \\
4\end{array}$ & 250.19 & $\begin{array}{c}120.0 \\
9\end{array}$ & $\begin{array}{c}120.0 \\
9\end{array}$ & 170.13 & 200.15 \\
\hline 6. & $\begin{array}{l}\text { Mg hardness } \\
(\mathrm{mg} / \mathrm{l})\end{array}$ & $\begin{array}{c}389.8 \\
4\end{array}$ & $\begin{array}{c}429.9 \\
1\end{array}$ & 289.80 & $\begin{array}{c}279.8 \\
4\end{array}$ & $\begin{array}{c}289.8 \\
8\end{array}$ & $\begin{array}{c}309.8 \\
8\end{array}$ & $\begin{array}{c}249.9 \\
0\end{array}$ & $\begin{array}{c}169.9 \\
0\end{array}$ & $\begin{array}{c}269.9 \\
2\end{array}$ & $\begin{array}{c}259.9 \\
0\end{array}$ & $\begin{array}{c}199.8 \\
8\end{array}$ & $\begin{array}{c}349.8 \\
8\end{array}$ & $\begin{array}{c}229.8 \\
8\end{array}$ & $\begin{array}{c}229.8 \\
9\end{array}$ & $\begin{array}{c}349.8 \\
2\end{array}$ & $\begin{array}{c}259.8 \\
5\end{array}$ & 469.80 & $\begin{array}{c}299.9 \\
0\end{array}$ & $\begin{array}{c}209.9 \\
0\end{array}$ & 359.86 & 419.84 \\
\hline 7. & $\begin{array}{l}\text { Total hardness } \\
(\mathrm{mg} / \mathrm{l})\end{array}$ & 580 & 580 & 530 & 470 & 440 & 460 & 370 & 290 & 370 & 380 & 340 & 490 & 370 & 360 & 570 & 440 & 720 & 420 & 330 & 530 & 620 \\
\hline 8. & & & & & & & & & & & & & & & & & & & & & & \\
\hline 9. & $\mathrm{Na}(\mathrm{meq} / \mathrm{l})$ & 5.3 & 1.2 & 4.6 & 3.9 & 1.8 & 2.4 & 1.4 & 1.6 & 3 & 4.3 & 2.9 & 4.1 & 1.9 & 2.4 & 9.5 & 5.2 & 11.8 & 3.3 & 3 & 13.4 & 15.2 \\
\hline 10. & $\mathrm{~K}(\mathrm{meq} / \mathrm{l})$ & 2.2 & 2.2 & 1.8 & 1.2 & 0.7 & 0.4 & 0.4 & 0.4 & 0.3 & 0.3 & 0.4 & 0.6 & 0.4 & 0.5 & 1 & 0.5 & 2.8 & 0.5 & 0.4 & 1.5 & 3.9 \\
\hline 11. & Free $\mathrm{CO}_{2}(\mathrm{mg} / \mathrm{l})$ & 72.6 & 39.6 & 59.4 & 48.4 & 19.8 & 41.8 & 30.8 & 17.6 & 17.6 & 37.4 & 22 & 30.8 & 24.2 & 28.6 & 77 & 28.6 & 101.2 & 26.4 & 24.2 & 70.4 & 79.2 \\
\hline 12. & Total acidity (mg & 107.5 & 45 & 87.5 & 85 & 55 & 47.5 & 37.5 & 30 & 30 & 50 & 25 & 52.5 & 45 & 115 & 125 & 115 & 117.5 & 57.5 & 37.5 & 117.5 & 132.5 \\
\hline 13. & $\mathrm{NO}_{3}(\mathrm{mg} / \mathrm{l})$ & 0.69 & 0.58 & 0.68 & 0.55 & 0.63 & 0.55 & 0.65 & 0.56 & 0.69 & 0.53 & 0.63 & 0.55 & 0.66 & 0.52 & 0.69 & 0.69 & 0.65 & 0.56 & 0.71 & 0.53 & 0.68 \\
\hline 14. & $\mathrm{HCO}_{3}(\mathrm{mg} / \mathrm{l})$ & $\begin{array}{c}119.5 \\
9\end{array}$ & $\begin{array}{c}104.9 \\
4\end{array}$ & 112.27 & $\begin{array}{c}101.2 \\
8\end{array}$ & 92.74 & 87.86 & 69.55 & 58.57 & 57.35 & 73.22 & 54.91 & 75.66 & 67.11 & 87.86 & $\begin{array}{c}141.5 \\
5\end{array}$ & 86.64 & 133.01 & 91.52 & 65.89 & 45.15 & 129.35 \\
\hline 15. & $\mathrm{CO}_{3}(\mathrm{mg} / \mathrm{l})$ & 0 & 0 & 0 & 0 & 0 & 0 & 0 & 0 & 0 & 0 & 0 & 0 & 0 & 0 & 0 & 0 & 0 & 0 & 0 & 0 & 0 \\
\hline 16. & Alkalinity (mg/l) & 490 & 430 & 460 & 415 & 380 & 360 & 285 & 240 & 235 & 300 & 225 & 310 & 275 & 360 & 580 & 355 & 545 & 375 & 270 & 185 & 530 \\
\hline 17. & $\begin{array}{l}\text { Turbidity } \\
\text { ( NTU) }\end{array}$ & 6 & 1 & 21 & 5 & 6 & 1 & 1 & 1 & 1 & 6 & 9 & 1 & 1 & 5 & 1 & 1 & 2 & 1 & 1 & 25 & 14 \\
\hline 18. & WQI & 65.98 & 32.58 & 213.79 & 50.30 & 62.32 & 15.26 & 15.45 & 9.22 & 18.94 & 58.88 & 95.18 & 20.62 & 17.51 & 50.34 & 19.09 & 18.51 & 35.93 & 19.88 & 18.46 & 245.71 & 143.86 \\
\hline
\end{tabular}


Table.5 Area wise distribution of various parameters for pre-monsoon period

\begin{tabular}{|c|c|c|c|c|}
\hline \multirow{2}{*}{ Parameter } & \multicolumn{2}{|c|}{ Classes } & \multirow{2}{*}{ Area $\left(\mathbf{k m}^{\mathbf{2}}\right)$} & Percentage area (\%) \\
\cline { 2 - 5 } & From & To & & \\
\hline \multirow{2}{*}{ TDS (mg/l) } & 180 & 500 & 32.092 & 98.593 \\
\cline { 2 - 5 } & 500 & 611 & 0.457 & 1.406 \\
\hline \multirow{2}{*}{ Ca content (mg/l) } & 40 & 75 & 29.933 & 91.959 \\
\cline { 2 - 5 } & 75 & 100 & 2.617 & 8.040 \\
\hline \multirow{2}{*}{ Mg content (mg/l) } & 40 & 100 & 32.193 & 98.902 \\
\cline { 2 - 5 } & 100 & 115 & 0.357 & 1.097 \\
\hline \multirow{2}{*}{ WQI } & 0 & 25 & 4.582 & 14.076 \\
\cline { 2 - 5 } & 25 & 50 & 18.240 & 56.036 \\
\cline { 2 - 5 } & 50 & 75 & 6.330 & 4.444 \\
\cline { 2 - 5 } & 75 & 100 & 1.506 & 5.808 \\
\hline
\end{tabular}

Fig. 1 Location map of sampling sites

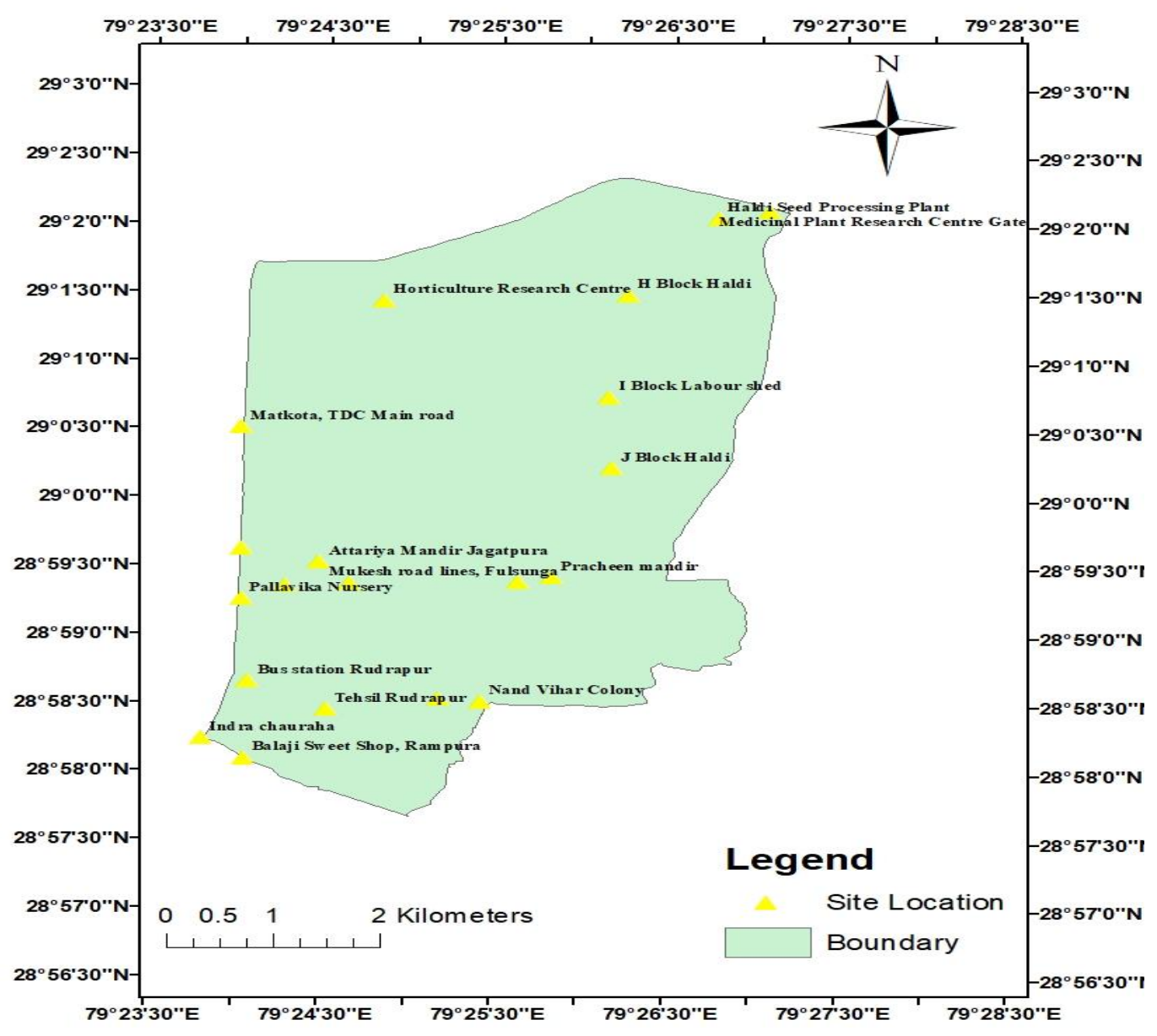


Fig.2 Flow chart for generation of WQI

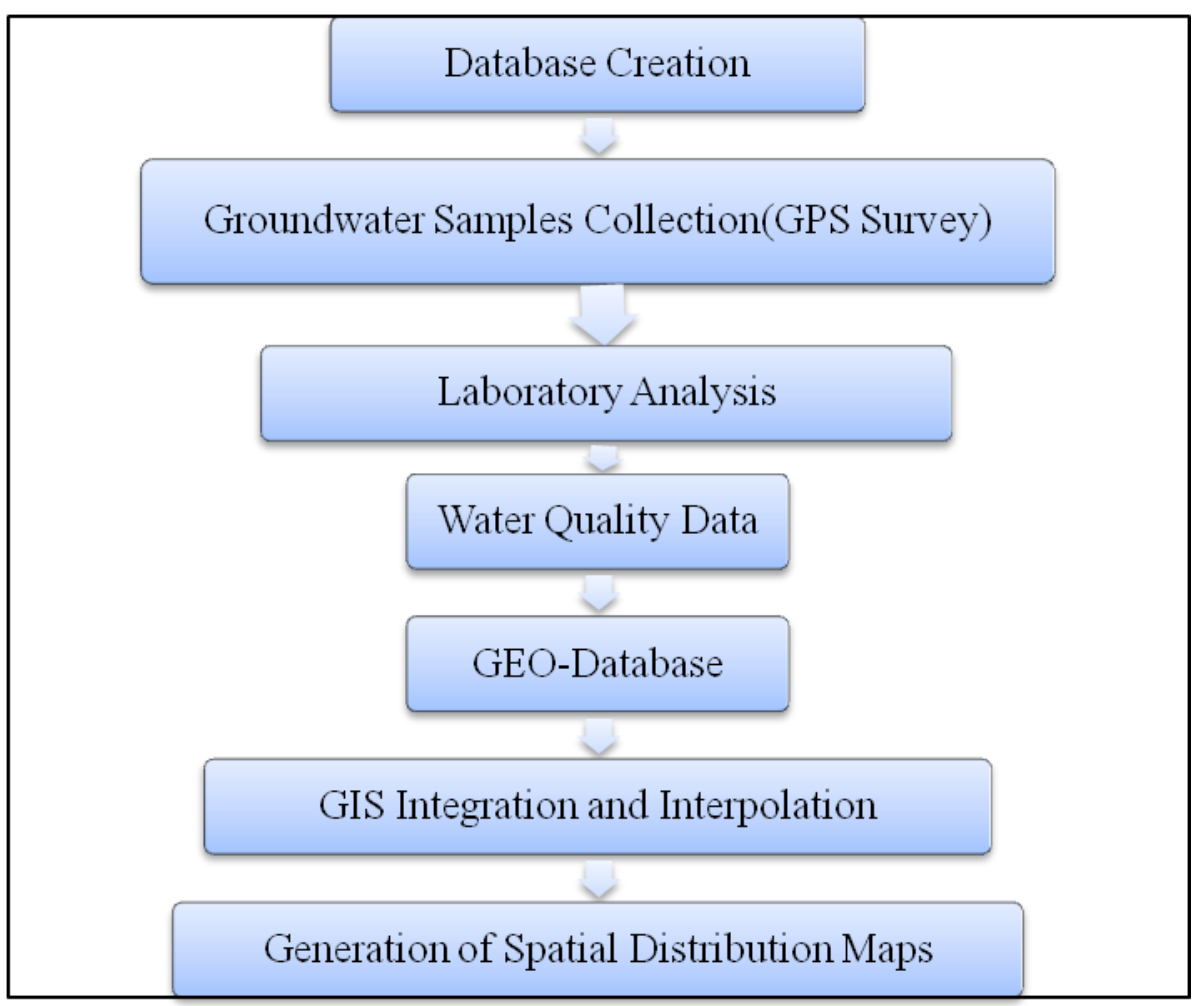

Fig. 3 Flow chart for generation of Water Quality Map

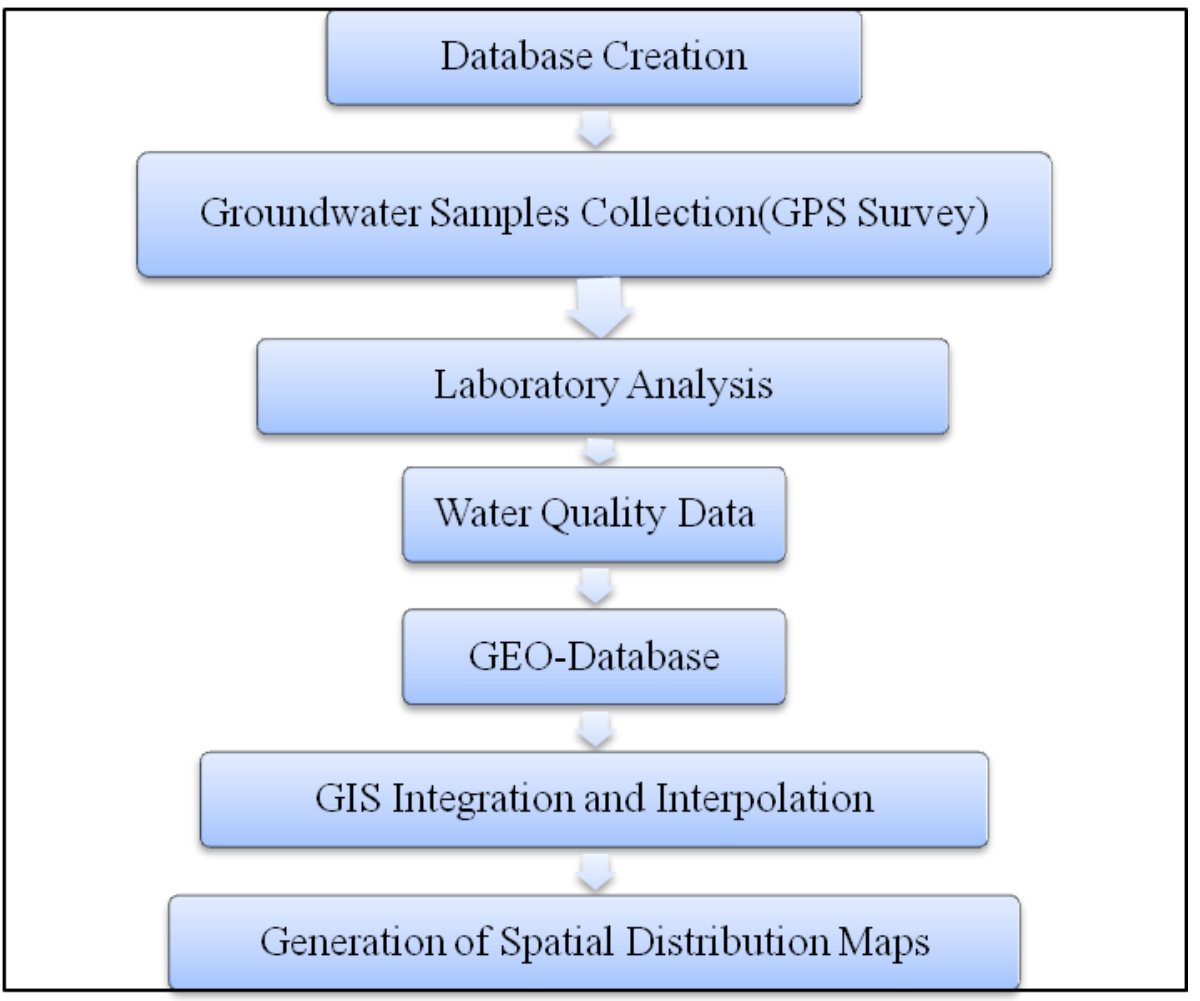


Fig. 4 Flow chart for generation of Water Quality Index map

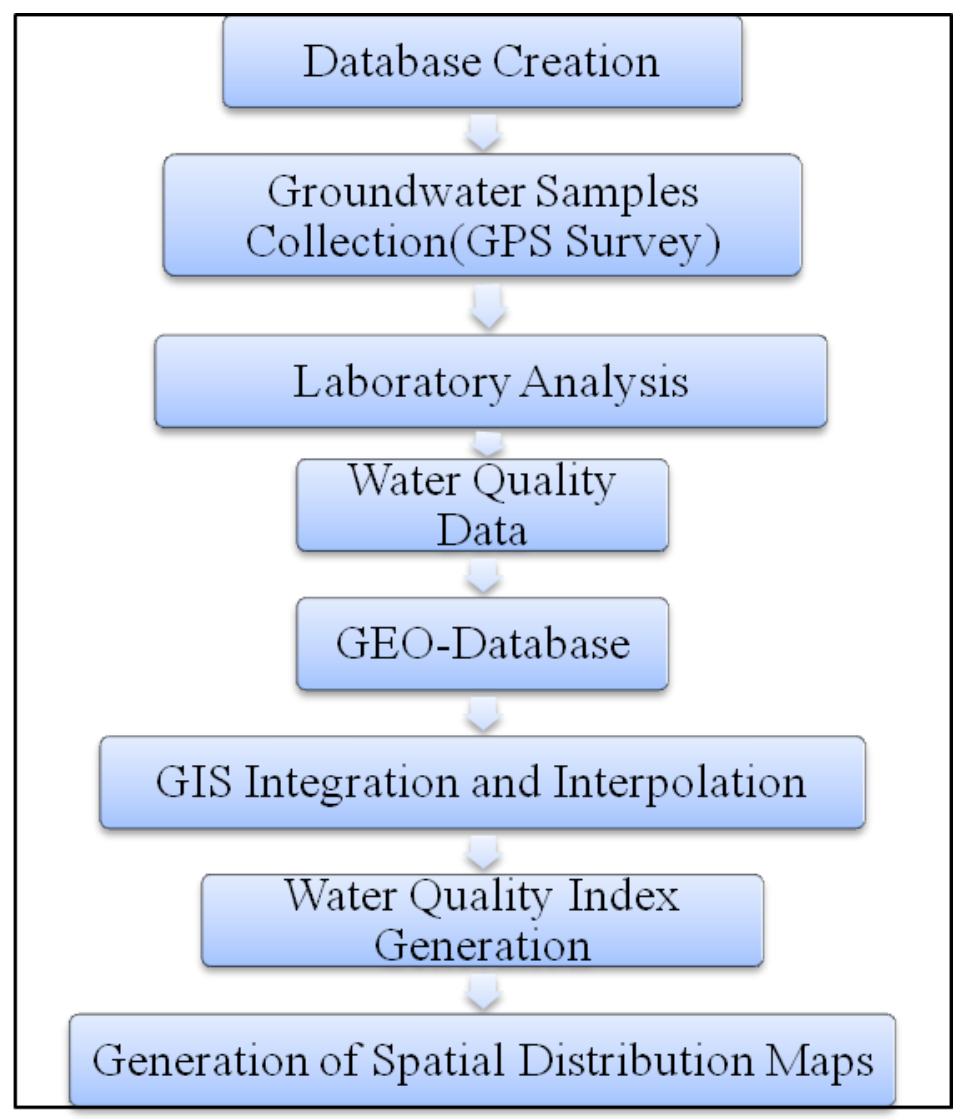

Fig. 5 TDS map for pre-monsoon period

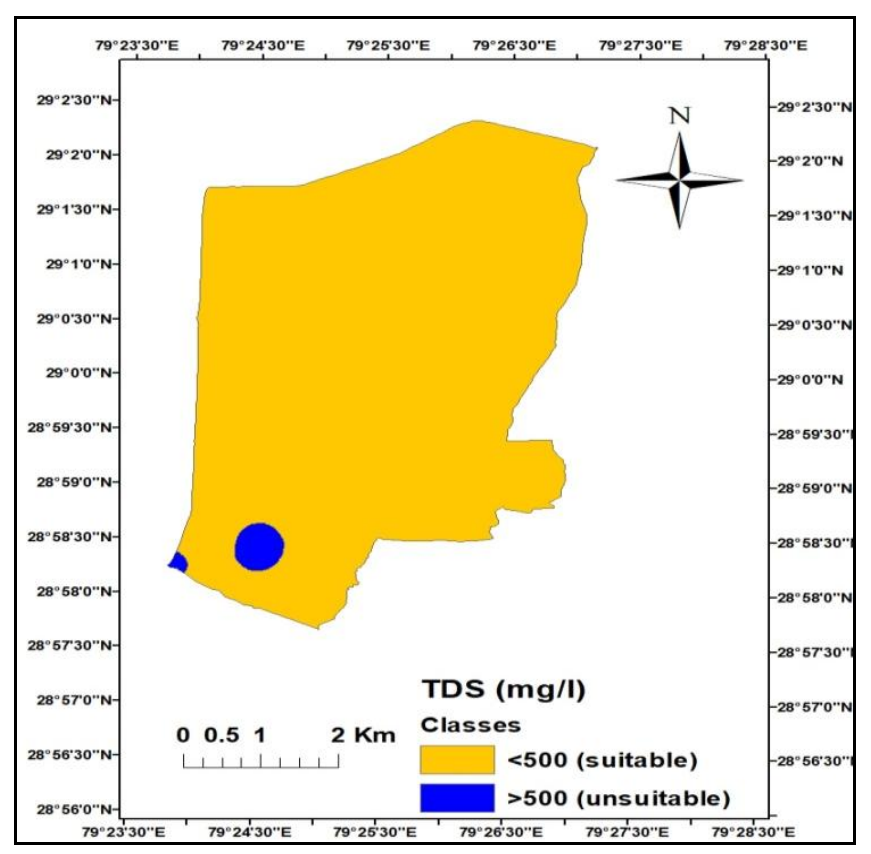


Fig. 6 Ca content map for pre-monsoon period

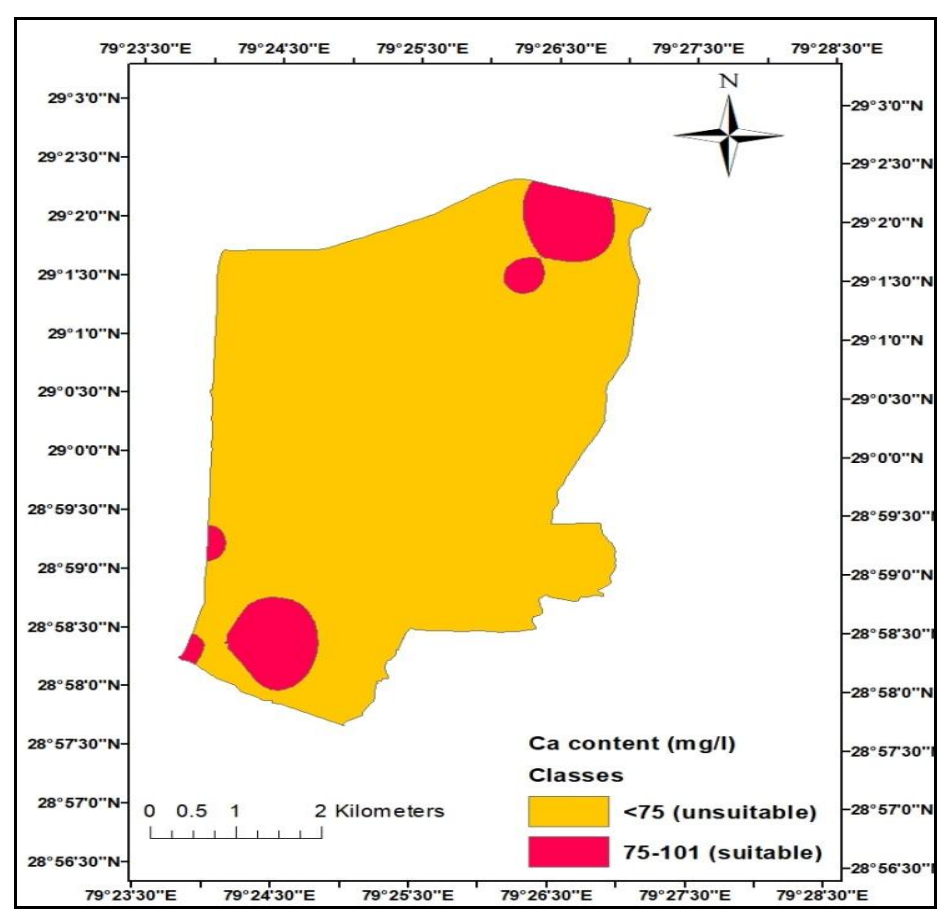

Fig. $7 \mathrm{Mg}$ content map for pre-monsoon period

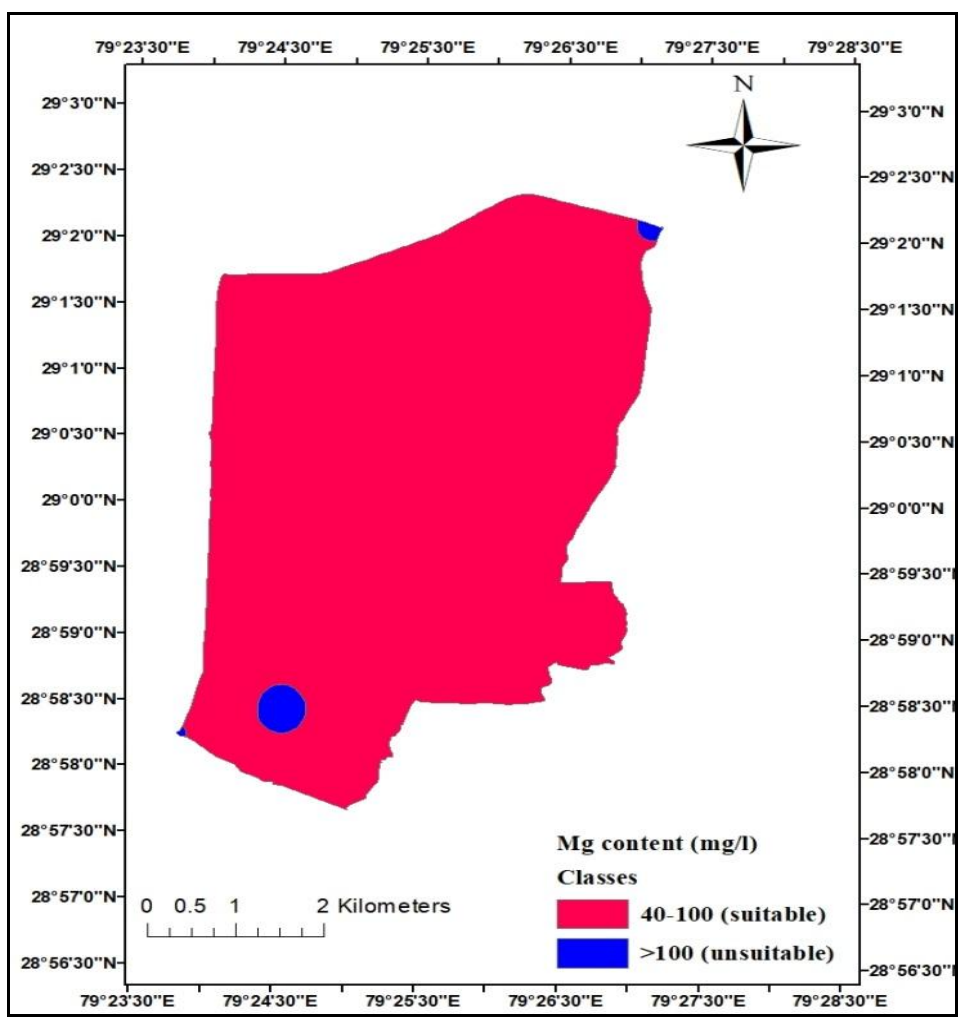


Fig. 8 WQI map for pre-monsoon period

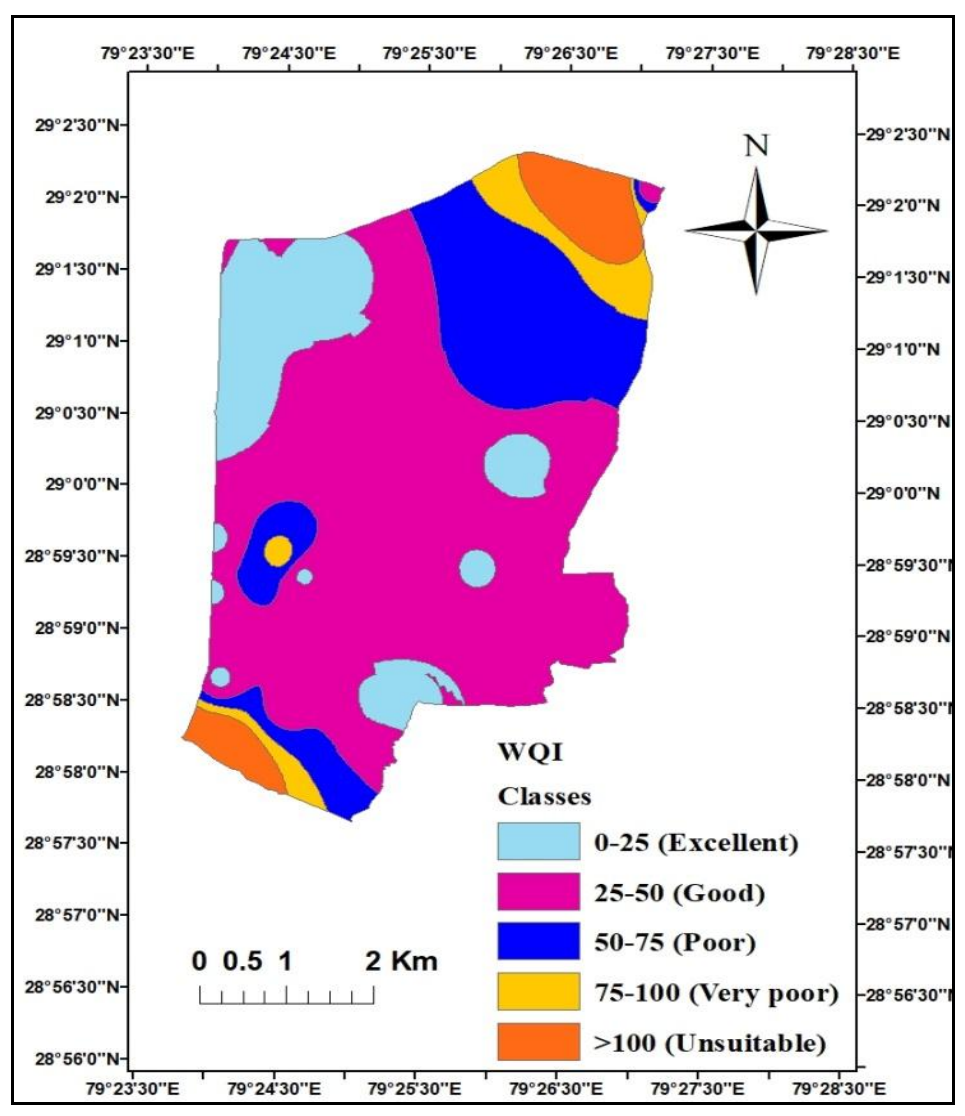

The physcio-chemical properties analysis of the Ground water samples were assessed for drinking purposes with the help of criteria given by Bureau of Indian Standards. Spatial distribution thematic maps of groundwater quality parameters and WQI were prepared under the GIS environment for drinking purpose. On the basis of above study the following conclusions were drawn:

$\mathrm{pH}$ value in all the samples analyzed except the sample nearby Chief Medical Officer Office road, Nand Vihar Colony, Model Medicinal Plants Garden, Pracheen Van Shakti and Haldi Attariya Mandir were found below the BIS permissible limit of $\mathrm{pH}$ (6.5-8.5) and were considered unsuitable for drinking purpose.

TDS value in all the samples analyzed except opposite to Tehsil, Gangapur road,
Indra chauraha were found within the BIS permissible limit of TDS $(<500$ $\mathrm{mg} / \mathrm{l}$ ) and were considered suitable for drinking purpose.

WQI analysis revels that area of $4.58 \mathrm{~km}^{2}$ with $14.07 \%$ was found excellent and area of $18.24 \mathrm{~km}^{2}$ with $56.03 \%$ was found under good category for drinking purpose.

\section{References}

A.O.A.C. 1950. Official and Tentative Methods of Analysis, The Association of Official Agricultural Chemists, Washington. Ed. 7:910.

Abbasi, T., Abbasi, S.A., 2012. Water quality indices. Elsevier, Amsterdam, Netherlands, pp. 384.

Alam, M., Rais, S. and Aslam, M. 2009.Hydro-chemical survey of 
groundwater of Delhi, India.E-Journal of Chemistry, 6(2):429-436.

Balan, I., Shivakumar, M. and Kumar, Pd. (2012). An assessment of ground water quality using water quality index in Chennai Tamil Nadu, India. Chronicles Young Scient.. 3:146-150.

Chauhan, A. and Singh, S. 2010. Evaluation of Ganga water for drinking purpose by water quality index at Rishikesh, Uttarakhand, India. Report Opinion, 2(9): 53-61.

Cheng, K.L. and Bray, R.H. 1951. Determination of calcium and magnesium in soil and plant material. Soil Science, 72:449-458.

Chowdhury, R.M., Muntasir, S.Y. and Monowar, H.M., 2012. Water Quality Index of Water Bodies Along Faridpur-Barisal Road in Bangladesh. Global Eng. Technol. Rev. 2(3): 1-8.

Jackson, M.L. 1973. Soil Chemical Analysis Prentice Hall of India Private Limited,
New Delhi.

Munger, J.R., Nippier, R.W. and Ingold, R.S. 1950. Analytical chemistry of the sulphur acids. Anal. Chem., 22:1455.

Singh, T. and Kalra, Y.P. 1975. Specific conductance method for in situ estimation of total dissolved solids. Journal of American Water Works Association, 67(2): 99.

Standford, S. and English, L. 1949. Use of the flame photometer in rapid soil tests for $\mathrm{K}$ and Ca. Agron. Journal, 4:446447.

Toth, S.J., Prince, A.I., Wallace, A. and Mikkelsen, D.S. 1948. Rapid quantitative determination of eight mineral elements in plant tissue by a systematic procedure involving use of a flame photometer. Soil Science, 66:459-466.

Wilcox, L.V. 1955. Classification and use of irrigation waters. USDA Criteria. Washington, D.C.

\section{How to cite this article:}

Dheeraj Kumar, Yogendra Kumar, Mohan lal, Mahima Bora, Sakshi Gautam and Sidra Souban. 2020. GIS Technique Based Spatio Temporal Variation Study of Ground Water Quality Parameters of SIDCUL-Pantnagar, India. Int.J.Curr.Microbiol.App.Sci. 9(04): 24412453. doi: https://doi.org/10.20546/ijcmas.2020.904.293 